\title{
ANOTHER PROOF OF ZHANG'S CONGRUENCE FOR THE EULER NUMBERS
}

\author{
SUMIT KUMAR JHA
}

\begin{abstract}
We give a proof of Zhang's congruence for the Euler numbers. The proof uses an explicit formula for the Euler numbers in terms of the Stirling numbers of the second kind.
\end{abstract}

Definition 1. The Euler numbers are a sequence of integers, denoted by $E_{n}$, which can be defined by the following Taylor series expansion

$$
\frac{1}{\cosh t}=\frac{2}{e^{t}+e^{-t}}=\sum_{n=0}^{\infty} \frac{E_{n}}{n !} \cdot t^{n},
$$

where $\cosh t$ is the hyperbolic cosine.

Zhang [1] proved the following

Theorem 1. We have, for all odd prime $p$,

$$
(-1)^{\frac{p-1}{2}} E_{p-1} \equiv-1+(-1)^{\frac{p-1}{2}} \quad(\bmod p) .
$$

Above result was proved in [1] by studying the calculating problem of the summation involving the Euler numbers, that is,

$$
\sum_{a_{1}+a_{2}+\cdots+a_{n}=k} \frac{E_{2 a_{1}} E_{2 a_{2}} \cdot E_{2 a_{k}}}{\left(2 a_{1}\right) !\left(2 a_{2}\right) ! \cdots\left(2 a_{k}\right) !}
$$

where the summation is over all $k$-dimension nonnegative integer coefficients $\left(a_{1}, a_{2}, \cdots, a_{k}\right)$ such that $a_{1}+a_{2}+\cdots+a_{k}=n$, and $k$ is any odd number with $k>1$.

We prove Theorem 1 using the following explicit formula for $E_{r}$, where $r$ is even.

Lemma 1. We have, for every even $r$,

$$
E_{r}=2 \sum_{k=1}^{r}(-1)^{k} \cdot k ! \cdot S(r, k)\left(\frac{1+i}{2}\right)^{k+1},
$$

where $i=\sqrt{-1}$, and $S(r, k)$ represents Stirling numbers of the second kind.

2010 Mathematics Subject Classification. 11B68.

Key words and phrases. Euler numbers, Zhang's congruence. 
Proof. Let $A_{r}$ be the number of alternating permutations of the set $\{1,2, \cdots, r\}$. (Let $S_{n}$ be the symmetric group of all permutations of $[n]:=\{1,2 \cdots, n\}$. A permutation $w=a_{1} a_{2} \cdots a_{n} \in S_{n}$ is called alternating if $a_{1}>a_{2}<a_{3}>a_{4}<$ $\cdots$. In other words, $a_{i}<a_{i+1}$ for $i$ even, and $a_{i}>a_{i+1}$ for $i$ odd). The formula (2) is a consequence of the following explicit formula for $A_{r}$

$$
A_{r}=2 i^{r+1} \mathbf{L} \mathbf{i}_{-r}(-i)=i^{r} \sum_{k=1}^{r} \frac{(-1)^{k} \cdot k ! \cdot S(r, k)}{2^{k}}(i+1)^{k+1},
$$

where $\mathbf{L} \mathbf{i}_{-r}(\cdot)$ is negative polylogarithm function, and $S(r, k)$ denotes the Stirling number of the second kind.

When $r$ is even, we have, $A_{r}=(-1)^{\frac{r}{2}} E_{r}$. We, now, proceed to prove the above result.

The polylogarithm function $\mathbf{L i}_{s}(z)$ is defined by the following power series in $z$

$$
\mathbf{L i}_{s}(z)=\sum_{k=1}^{\infty} \frac{z^{k}}{k^{s}}
$$

where $|z|<1$. We know that, from [2],

$$
\mathbf{L i}_{s}(-i)=-2^{-s} \eta(s)-i \beta(s)
$$

where $\eta(s)$ and $\beta(s)$ are the Dirichlet eta and beta functions, respectively. Letting, $s=-r$, a negative integer in the above relation, we get

$$
\mathbf{L i}_{-r}(-i)=-2^{-s} \eta(-r)-i \beta(-r)=-\left(2^{r} \frac{2^{r+1}-1}{r+1} B_{r+1}+i \frac{E_{r}}{2}\right),
$$

where $B_{r+1}$ and $E_{r}$ represent Bernoulli and Euler numbers, respectively.

We conclude our result (3) after the following fact, from [3],

$$
A_{r}=\left\{\begin{array}{ll}
(-1)^{\frac{r-1}{2}} \frac{2^{r+1}\left(2^{r+1}-1\right) B_{r+1}}{r+1} & \text { when } \mathrm{r} \text { is odd } \\
(-1)^{\frac{r}{2}} E_{r} & \text { when } \mathrm{r} \text { is even }
\end{array},\right.
$$

and the following representation from the note [4]

$$
\mathbf{L i}_{-r}(-x)=\sum_{k=1}^{r} k ! S(r, k)\left(\frac{1}{1+x}\right)^{k+1}(-x)^{k} .
$$

We would also require the following congruence involving Stirling numbers of the second kind

Lemma 2. We have, for all odd prime $p$,

$$
(-1)^{k} \cdot k ! \cdot S(p-1, k) \equiv-1 \quad(\bmod p),
$$

for $1 \leq k \leq p-1$. 
Proof. We have the following explicit formula for Stirling numbers of the second kind [5]

$$
S(p-1, k)=\frac{1}{k !} \sum_{j=1}^{k}(-1)^{k-j}\left(\begin{array}{l}
k \\
j
\end{array}\right) j^{p-1} .
$$

Using the above, and Fermat's little theorem we can conclude our result.

Proof of Theorem 1. Letting $r=p-1$, where $p$ is an odd prime, in equation (2) gives us

$$
E_{p-1}=2 \sum_{k=1}^{p-1}(-1)^{k} \cdot k ! \cdot S(p-1, k)\left(\frac{1+i}{2}\right)^{k+1}
$$

Considering the fact that $2^{p-1} \equiv 1(\bmod p)$, and Lemma 2 , we have,

$$
\begin{aligned}
& E_{p-1} \equiv 2^{p-1} E_{p-1} \equiv-\sum_{k=1}^{p-1} 2^{p} \cdot\left(\frac{1+i}{2}\right)^{k+1} \quad(\bmod p) \\
& \equiv-2^{p} \cdot\left(\frac{-1}{2}+2^{-\frac{p}{2}} \sin \left(\frac{\pi p}{4}\right)+i\left(\frac{1}{2}-2^{-p / 2} \cos \left(\frac{\pi p}{4}\right)\right)\right) \quad(\bmod p) .
\end{aligned}
$$

Now, discarding the imaginary part in the above, since, $E_{p-1}$ are integers, we get

$$
\begin{aligned}
& E_{p-1} \equiv-2^{p} \cdot\left(\frac{-1}{2}+2^{-\frac{p}{2}} \sin \left(\frac{\pi p}{4}\right)\right) \quad(\bmod p) \\
& \equiv 2^{p-1}-2^{\frac{p-1}{2}} \cdot \sqrt{2} \cdot \sin \left(\frac{\pi p}{4}\right) \quad(\bmod p) .
\end{aligned}
$$

But, we have

$$
2^{\frac{p-1}{2}} \equiv\left(\frac{2}{p}\right) \equiv(-1)^{\frac{p^{2}-1}{8}} \quad(\bmod p)
$$

where $\left(\frac{a}{b}\right)$ represents the Legendre's symbol. As a result, we can conclude that

$$
E_{p-1} \equiv 1-(-1)^{\frac{p^{2}-1}{8}} \cdot \sqrt{2} \sin \left(\frac{\pi p}{4}\right) \quad(\bmod p) .
$$

Now, since $\sqrt{2} \sin \left(\frac{\pi p}{4}\right)=(-1)^{\frac{-(p-1)(p-3)}{8}}$, we have

$$
E_{p-1} \equiv 1+(-1)^{\frac{p+1}{2}} \quad(\bmod p)
$$

which is our result Theorem 1. 


\section{REFERENCES}

1. Zhang, W.P. (1998). Some identities involving the Euler and the central factorial numbers. Fibonacci Quaterly. 36 (4): 154-157.

2. Prudnikov, A. P.; Marichev, O. I.; and Brychkov, Yu. A. The Generalized Zeta Function $\zeta(s, x)$, Bernoulli Polynomials $B_{n}(x)$, Euler Polynomials $E_{n}(x)$, and Polylogarithms $L i_{\nu}(x)$. 1.2 in Integrals and Series, Vol. 3: More Special Functions. Newark, NJ: Gordon and Breach, pp. 23-24, 1990.

3. Stanley, Richard P. A survey of alternating permutations. Contemp. Math 531 (2010): 165-196.

4. S. E. Landsburg, Stirling numbers and polylogarithms, unpublished note, 2009, http://www.landsburg.com/query.pdf.

5. Dilcher, K. (2000). An Extension of Fermats Little Theorem, and Congruences for Stirling Numbers. The American Mathematical Monthly, 107(10), 936. doi:10.2307/2695589

International Institute of Information Technology, Hyderabad, India

E-mail address: kumarjha.sumit@research.iiit.ac.in 